\title{
Modeling Electricity Consumption using Modified Newton's Method
}

\author{
P. Ozoh \\ Faculty of Computer \\ Science \&Information \\ Technology, Universiti \\ Malaysia, \\ Sarawak
}

\author{
S. Abd-Rahman \\ Faculty of Computer \\ Science\& Information \\ Technology, Universiti \\ Malaysia, \\ Sarawak
}

\author{
J. Labadin \\ Faculty of Computer \\ \& Information \\ Technology, Universiti \\ Malaysia, \\ Sarawak
}

\author{
M. Apperley \\ Department of Computer \\ Science \\ University of Waikato \\ New Zealand
}

\begin{abstract}
In this paper we present modified Newton's model (MNM) to model electricity consumption data. A previous method to model electricity consumption data was done using forecasting technique (FT) and artificial neural networks (ANN). A drawback to previous techniques is that computations give less reliable results when compared to MNM. A comparative analysis is carried out for FT, ANN and MNM to investigate which of these methods is the most reliable technique. The results indicate that MNM model reduced mean absolute percentage error (MAPE) to $0.93 \%$, while those of FT and ANN were $3.01 \%$ and $3.11 \%$, respectively. Based on these error measures, the study shows that the three methods are highly accurate modeling techniques, but MNM was found to be the best technique when mining information. Experimental results indicate that MNM is the most accurate when compared to FT and ANN and thus has the best competitive performance level.
\end{abstract}

\section{Keywords}

Efficiency, modified newton's method, forecasting technique, artificial neural networks, reliability

\section{INTRODUCTION}

Growing demand for electricity consumption makes it imperative to strengthen capabilities in infrastructure and equipments. Energy efficiency is becoming an interesting area of research because there are constraints in terms of availability of electricity supply and distribution, hence the needs for efficiency in its use [1] . For this reason, energy analysts need a guideline to better choose the most appropriate technique in order to provide accurate estimates for electricity consumption problems. FT, as used in previous literature [1], [2], consists of a combination of time series, ARIMA and forecasting techniques to solve energy models. The research papers carry out a comprehensive review and computes different evaluation statistics for these techniques. It is discovered that the approach to the various techniques involved in FT are similar in their analytical solution process.

The problems entrenched in electric-efficient systems are diverse. Whereas electric generation, distribution and consumption have greatly improved in the society, efficient use of it is a growing problem. This has led to escalating costs for electricity consumption [3]. A novel regression technique, evolution local kernel regression (ELKR) was introduced by [4]. It is a kernel regression variant based on local NadarayaWatson estimates with independent bandwidths distributed in data space. The model uses a stochastic non-convex optimization method known as CMA-ES. The constraints in the research work consist of absence of parameter studies of (ELKR) on real power data and also a lack of experiments in regression problems. [4] investigates half-hourly ahead load predicting and half-hourly ahead price prediction applications. The method used in the research paper is artificial neural network (ANN) which uses historical data, load consumption, price and calendar data. It develops a multi-layer feed forward neural network trained with Back propagation (BP) algorithm. The technique considers only short-term load predicting or forecasting system. For an efficient electricity system, it is necessary to consider a long-term collection of data and also use of real-time data [5]. Proposed algorithms for minimization of the electric production costs of power is obtained by making a comparative study of genetic algorithms and Newton's method. The algorithms seem to be an effective technique in solving a great number of problems which are in constant evolution. However, there are limitations in the method. The technique does not present results of number of iterations involved in numerical solution of the problem for its rate of convergence, to determine its performance index. A simulation to model behavior of an electricity network, following the introduction of an electric vehicle fleet and high wind penetration, was described by [6] . In the vehicle model simulation, it takes into consideration the electrical model when vehicle is connected to the grid and the vehicle behavior model that determines timing and energy use of trips made by individual vehicles. It incorporates accurate wind speed data and measured corresponding grid load over a one-year period. A system to develop informed demand-side load management techniques to support and promote energy efficiency and economy in the residential/household sector was developed by [7]. The paper discusses the communication of real-time information to consumers through specific and ambient visualizations. A based game scenario that addresses the problem of energy consumption and possible learnability solutions is discussed by [8]. It proposes a novel way of using mobile Augmented Reality (AR) in combination with adaptive virtual gardens to raise awareness for reducing energy consumption through a game setup. Results from the study shows that the presented game motivates players to be aware of the presented problem.

The present study models historical electricity consumption data and utilizes Newton's method, which together with standard error estimates of data are used to obtain MNM. The study carries out a comparative analysis with a simulation of electricity consumption data based on FT in order choose a reliable method for modeling. Newton's method has previously been used to solve energy problems in the downlink of an orthogonal frequency division multiple accesses (OFDMA), [9]. It is applicable in the field of computational learning theory. The Newton's technique is very fast, considers multiple parameter constraint violations in its control task and possesses high speed computation [5]. 
This research work aims to estimate electric load in the system with the goal of modeling electricity consumption.

\section{METHODOLOGY}

This research is concerned with comparing solution techniques for modeling electricity consumption data. Efficient use of energy is becoming an important issue since the future world is dependent on today's decision. Identifying a reliable solution to electricity problems should help in achieving efficiency by the fact that forecasting for electricity consumption is done at a faster rate. The requirement for efficient use of electricity is proper allocation of available resources. This is made possible by accurate prediction of future needs, through its proper estimation process as shown in Figure 1. To determine the recommended technique for modeling electricity consumption data, an extensive review of literature was conducted on published literature related to the study. Specifically, the information compiled focuses on strengths and weaknesses of past techniques related to this study.

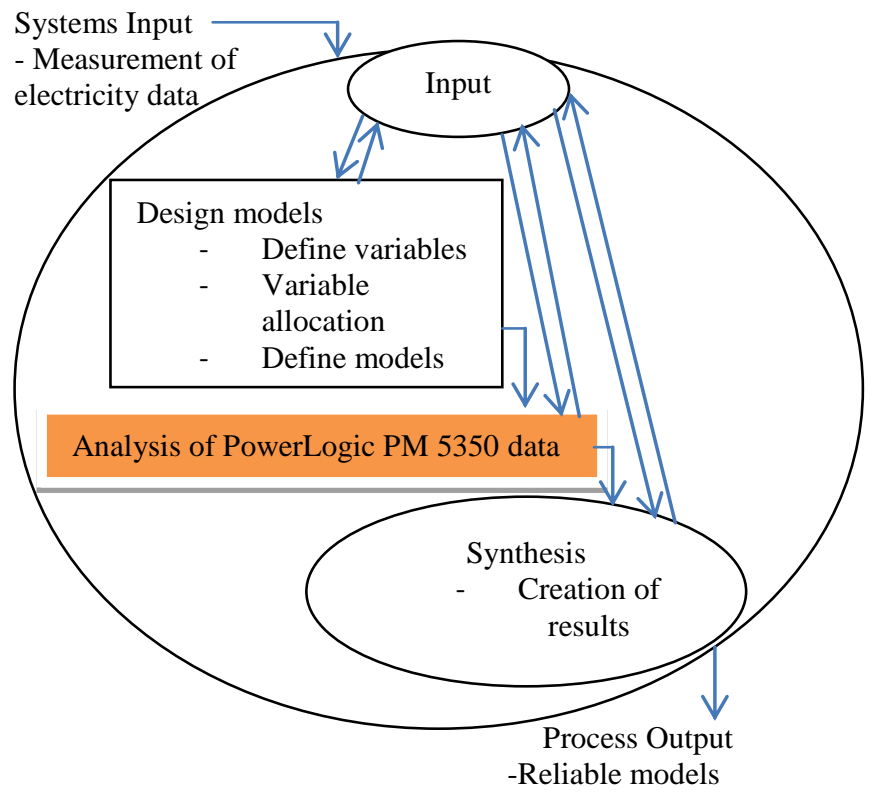

Fig. 1: Computation of estimates for electricity consumption

MNM was selected based on the fact that it has the most accurate results based on the techniques considered in this research and has a proven high performance level. In order to make comparisons between MNM, FT and ANN methods, there is a need to formulate respective models, simulated from electricity consumption data, $\mathrm{f}(\mathrm{x})$, where $\mathrm{x}=\mathrm{x}_{1}, \mathrm{x}_{2}, \mathrm{x}_{3}, \ldots \ldots \mathrm{x}_{\mathrm{n}}$ are occurrences at time $\mathrm{x}$.

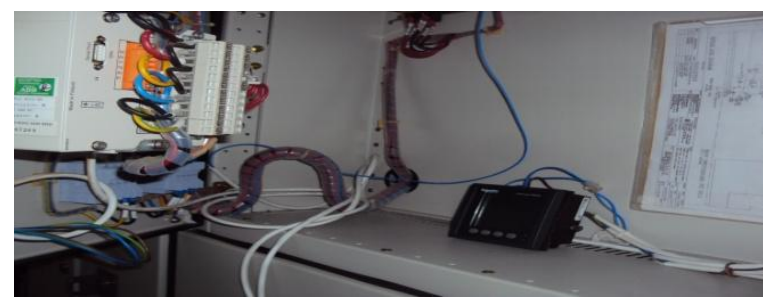

Fig. 2: The PowerLogic PM 5350

The electricity consumption data used in the analysis and computations of this research work consists of historical weekly aggregate power consumption data taken from daily recordings using the PowerLogic PM 5350, shown in Figure 2. The data samples were drawn from the Universiti Malaysia in Sarawak, Malaysia. Models obtained in this paper are applied in the power industry for forecasting electricity consumption. It should be noted that weekly consumption pattern can sometimes vary, depending on some factors such as a breakdown in the air-conditioner units, which results in a reduction in electricity consumption. For the estimation process, four years historical data i.e. a weekly aggregate of 1460 average daily data sets of the years 2009-2012 are used for training the forecasting models and the weeks in January and February 2013 month samples are used for testing these models. To illustrate the behavior of the propose technique, results comparing four weeks corresponding to the months of January and February for the previous years are presented. In this manner, representative results for this study are provided. For the sake of a fair comparison, four weeks in each of the months of January and February are selected. To build the forecasting models for each one of the considered weeks, the information available includes average daily consumption data of the weeks previous to the week whose data are to be predicted.

The FT method was evaluated using Box-Jenkins (BJ) approach. This is because the study considers univariate timeseries method, i.e. only weekly electricity consumption forecasts are required. This is because univariate methods predict the future value of a time series solely from its past historical data. The STEPWISE variable selection procedure, available on the SPSS for Windows 21.0 statistical package was used to obtain models. Once models are obtained, these are used as independent and input variables using multiple perception in ANN techniques. Forecasts were generated from Newton's method using MATLAB programming techniques, where prediction errors are computed and added to these forecasts to compute new forecasts for MNM method. Once forecasts were generated from these techniques, the mean absolute percentage error (MAPE) were computed and compared. Relative measures dealing with percentage errors were used to compare the ex post forecasting accuracy of the different techniques. Comparisons of results obtained from simulating electricity consumption data using MNM, ANN and FT methods with actual data are used to check for reliability and accuracy of the methods.

The following solution techniques were considered:

\subsection{Forecasting Technique}

In this research, modeling of electricity consumption data is undertaken using FT technique. A similar study to obtain forecast of energy consumption to promote sustainable energy development was carried out using a combination of grey and FT [10].

A forecasting technique relates $\mathrm{Y}$ to a function of $\mathrm{X}$ and $\beta$ and can be represented as:

$$
\mathrm{Y}_{\mathrm{n}} \approx \mathrm{f}\left(\mathrm{X}_{\mathrm{n}, \beta)},\right.
$$

where $Y_{n}$ consists electricity consumption data for a given week $n . X_{n}$ is weekly data bill for week $n$. $\beta$ is the unknown parameter. It has been applied to solve non-linear problems [11]. Hence, we obtain a model from given electricity consumption data bill. This would be used to predict weekly electricity consumption based on a 1460 daily set of data covering 4 years. 


\subsection{Artificial Neural Network}

Artificial neural networks (ANN) are mathematical models consisting of many simple processing units, called neurons that perform a local computation on their input to produce an output. Neural networks are similar to the least square estimation technique and can be viewed as an alternative statistical approach to solving least squares problems [12].

ANN training basically consists of determining the network parameters such as weights, scaling factors and others, that allow achieving the desired objective based on the available training sets. Training stops when performance has been minimized to the goal, the performance gradient falls below a minimum gradient, and the maximum amount of time has been exceeded.

\subsection{Modified Newton's method}

The proposed technique, MNM is used to predict new estimates for electricity consumption data and consists of a combination of Newton's method and standard error estimates. Newton's technique has previously been applied to wireless sensor network for enhancing efficiency in heating, ventilation and air-conditioning (HVAC) systems [8]. The MNM will at each time-step, evaluate the function to estimate first-order derivatives. It will use this information towards obtaining a model that fits electricity consumption data. The algorithm for analysis of Newton's algorithm is given in Figure 3.

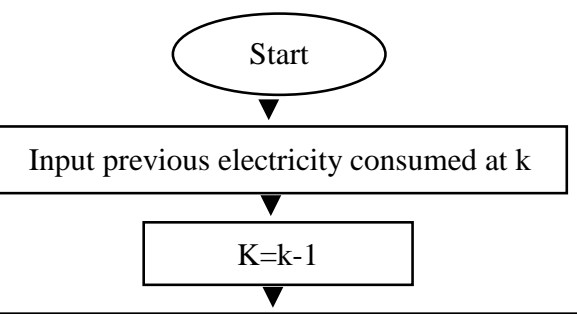

Evaluation generation of the adoptive function for electricity consumption

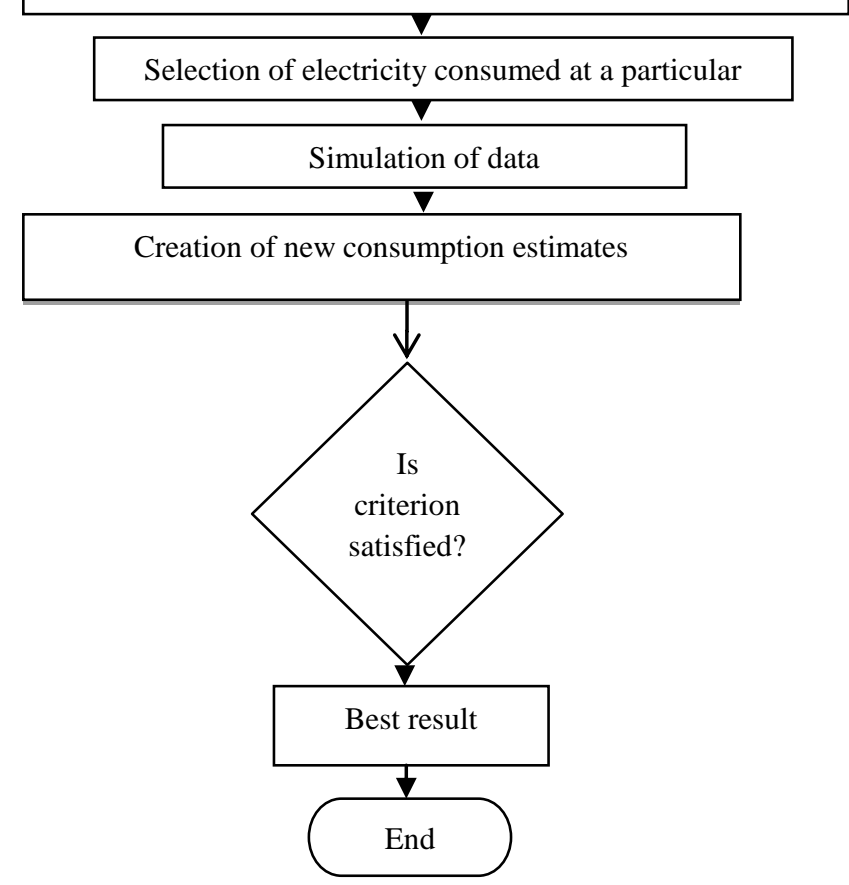

Fig. 3: The algorithm for Newton's method
MNM can be expressed as follows:

$$
\begin{aligned}
& x_{n+1}=x_{n}-\frac{f\left(x_{n}\right)}{f^{\prime}\left(x_{n}\right)}+S E \\
& S E=\sum_{t=1}^{n} \sqrt{\frac{\left(Y_{t}-X_{t}\right)^{2}}{n}}
\end{aligned}
$$

where $Y_{t}$ and $X_{t}$ are the the estimated and actual values respectively and $\mathrm{n}$ is the total number of data. Newton's method is an iterative and recursive technique that computes $\mathrm{x}_{\mathrm{n}}$ based on previous estimates $\mathrm{x}_{\mathrm{n}-1}, \mathrm{x}_{\mathrm{n}-2}, \ldots, \mathrm{x}_{\mathrm{n}-\mathrm{k}}$. It is approximated as a non-linear model.

\section{RESULTS AND DISCUSSIONS}

To get an idea about performance of MNM, ANN and FT, an independent set of simulation for historical electricity consumption data was conducted using each method and models obtained for them.

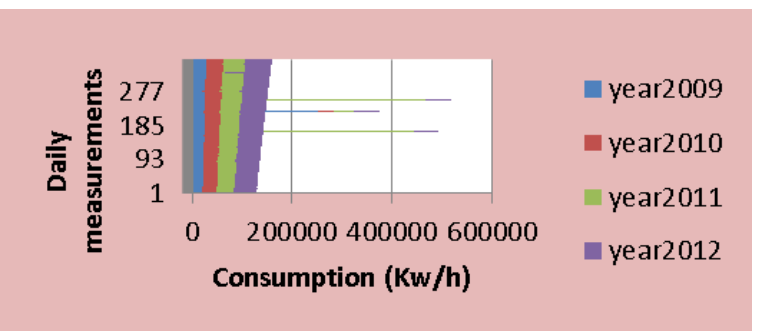

Fig. 4: Daily electricity consumed from JanuaryDecember between 2009-2012

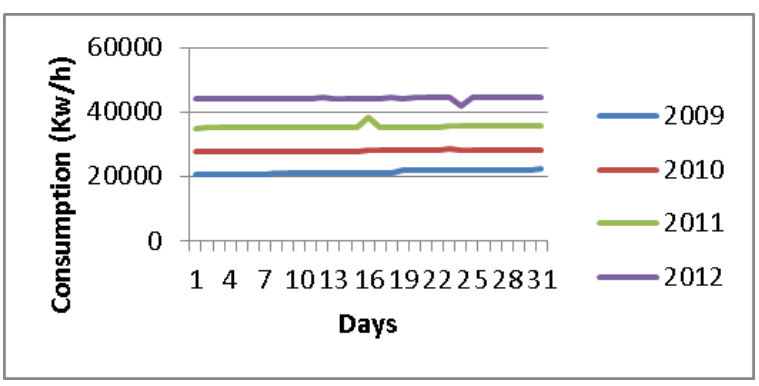

Fig. 5: Daily electricity consumption for January, 20092012

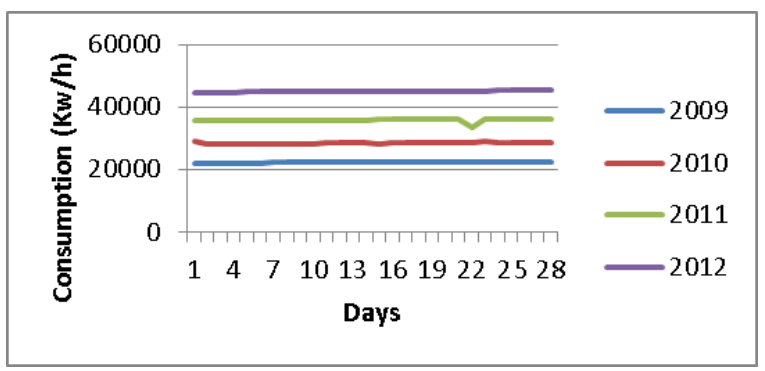

Fig. 6: Daily electricity consumption for February, 20092012

Figure 4 show that the electricity consumed has been following an increasing pattern through the years. The figure captures daily electricity consumption readings. Figure 5 and 
Figure 6, which are used as baselines for making predictions, indicate daily electricity consumption for January and February 2009-2012. Demand for electricity has had an upward trend. This makes it imperative for more efficient use of it and for the development of models that will assist in its efficient use.

Consumption models were first used to generate forecasts based on historical data-set. Following this procedure, relative measures of forecasting accuracy dealing with the mean absolute percentage error (MAPE) was applied to compare the performance of the models. This was previously applied in [29]. The measure used is explained as follows:

$$
\mathrm{MAPE}=\sum_{\mathrm{i}=1}^{\mathrm{n}} \frac{\left|\mathrm{PE}_{\mathrm{i}}\right|}{\mathrm{n}}
$$

$\left|\mathrm{PE}_{\mathrm{i}}\right|$ is the absolute value of the percentage error.

$$
P E_{t}=\left(A_{t}-F_{t}\right) / X_{t} \times 100 \%
$$

where $X_{t}$ is the actual value at period $t, F_{t}$ is the forecast value at period $t$ and $n$ is the number of forecasts.

The purpose of these relative errors is to determine the percentage error of the forecasts, i.e. the difference between the actual and forecast values, expressed as a percentage of the actual value, followed by finding the mean and mean absolute values of these percentage errors. The significance of these measures has been discussed in detail by [15], [28]. To summarize and compare the predictive ability of these three techniques, Table 1 shows the computed values of the relative measures of accuracy of the different forecasting techniques. The results of the comparative study showed that MNM technique had the lowest MAPE (0.93), and hence the most accurate. The FT technique was less accurate, obtaining a MAPE value of 3.01. The ANN was the most inaccurate method with the highest MAPE (3.11).

From the results obtained, the hybrid MNM model, which is a combination of Newton's method and the recursive corrector factor based on standard error estimates, gives better predictions than either FT or ANN forecasts and its overall forecasting capability is improved.

Table 1. Relative measure of the accuracy of different forecasting techniques

\begin{tabular}{|c|c|c|c|}
\hline Measure of accuracy & FT & ANN & MNM \\
\hline $\begin{array}{c}\text { Mean absolute percentage } \\
\text { Error (MAPE) }\end{array}$ & 3.01 & 3.11 & 0.93 \\
\hline
\end{tabular}

In this paper, several different approaches are used to investigate the most reliable method to predict electricity consumption data. Forecasting using MRM, ANN and MNM methods are computed for each week for the months under study. Models are obtained for each week of the months under consideration using historical data for 2009-2012. The computation of forecasting data is based on previous week's data for January and February 2012. In general, computations for weekly data for other years are based on data for previous years, i.e. forecasting is carried out recursively. The predicted weekly electricity consumption data for January and February 2009 to 2012 using the different techniques are given in Figure 7 and Figure 8. The figures shows the results of actual and predicted weekly aggregate of daily electricity consumption for the months of January 2013 and February 2013 by FT, ANN and MNM respectively.
It is clear from the results that predicted consumption patterns are very much similar to the actual one using MNM method, in comparison with other techniques. The superiority of the MNM technique in forecasting performance has been shown in the present study. This proposed technique out-performs traditional forecasting methods in predictive accuracy.

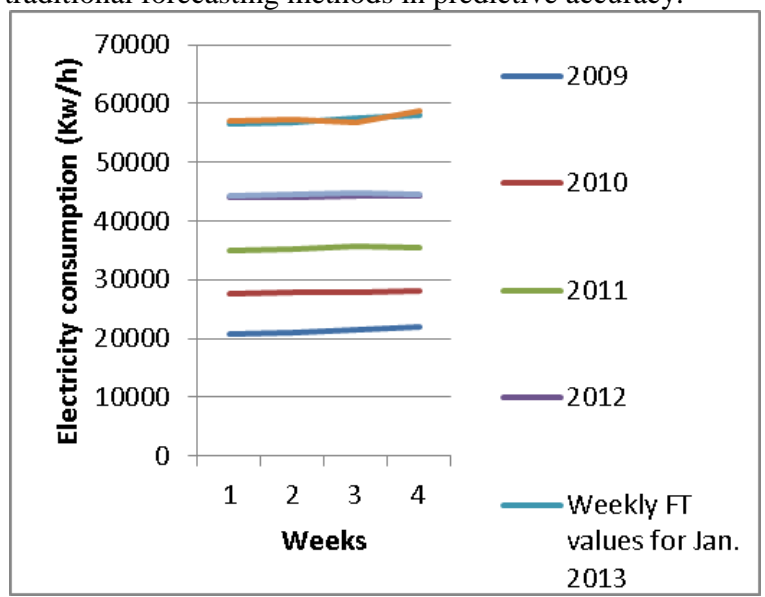

Fig. 7: Forecasting results of January 2013

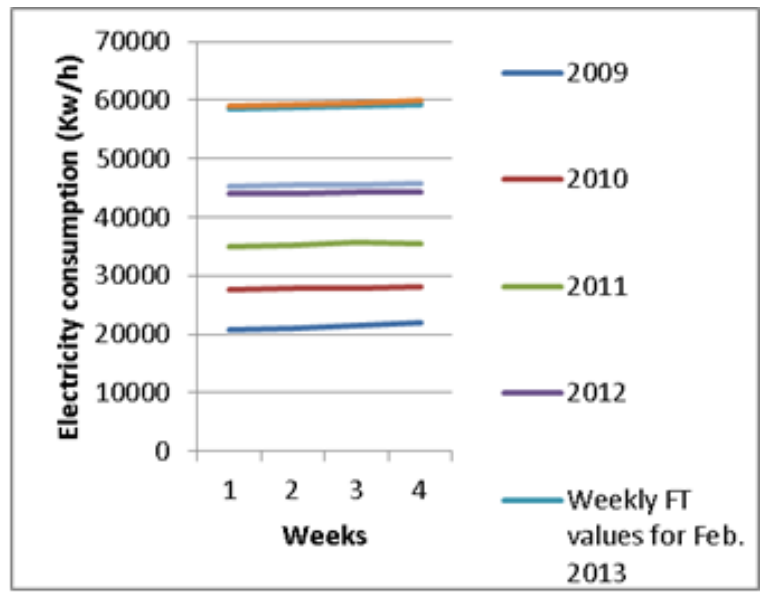

Fig. 8: Forecasting results of February 2013

\section{CONCLUSION AND FUTURE WORK}

Electricity consumption expenses are becoming an increasing important component in power management systems. In this paper, models are obtained from simulating electricity consumption data in order to discover a reliable technique for modeling such data.

Empirical studies have often compared the forecasting accuracy of time-series modeling approaches because of the different theoretical concepts of these techniques. This paper has extended the boundary to include the MNM technique which is based on a hybrid recursive correction method, and is a combination of Newton's method together with standard error estimates. The choice of forecasting technique becomes the important influence factors on how to improve electricity consumption forecasting accuracy. From the results discussed above, it is observed that the predicted electricity consumption data by MNM model are very much similar to the actual consumption data. The superiority of the MNM technique in forecasting is shown in its achieving the lowest MAPE value. This is because the MNM is designed to capture functional forms of automatically, allowing for non-linear 
relationships between the modeling variables in the timeseries data. Hence, we propose the error correction method for the generations of new models in which the new forecasts are suitable to train the MNM technique are generated by correcting the historical day's data with the help of consumption correction estimates. For learning the MNM method, the values obtained consists of correction and forecast errors. To verify the forecasting ability of the proposed method, we performed simulations for consumption forecasting by FT, ANN and MNM model approach. The test results showed that the proposed forecasting method could provide a considerable improvement of electricity consumption accuracy, especially as the MNM model gives better predictions than either FT or ANN forecasts, and its overall forecasting capability is improved. However, model specification and identification has always been a problem in modeling because human judgment is required for forming complex non-linear relationships. On the contrary, the power of the MNM exists in its inherent ability to successfully model and forecast complex non-linear systems. It identifies strengths in model evaluation, and is thus the most reliable method for modeling electricity consumption data.

Identifying a discrete time based model helps improve efficiency by making pre-dictions for electricity consumption to be faster and more reliable. The model accounts for this behavior by considering electricity consumption over a period of time. As a next step work, research involving applying control measures to low and high power appliances would be undertaken. It involves introducing power saving measures to low and high power appliances. In previous research, only HVAC systems were considered for control.

\section{ACKNOWLEDGEMENT}

Patrick Ozoh thanks Universiti Malaysia, Sarawak for the Zamalah research study award and the Tertiary Education Trust Fund (TETF) of the Nigerian government both of which have contributed to the development of this research work.

\section{REFERENCES}

[1] L. Sughnathi and A. A. Samuel, "Energy models for demand forecasting - A Review," Renew. Sustain. Energy Rev., vol. 16, pp. 1223-1240, 2012.
[2] C. D. Lewis, Industrial and Business Forecasting Methods: A practical guide to exponential smoothing and curve fitting, Butterworth Scientific, London, 1982.

[3] J.C. Lam, K. K. W. Wan, D. Liu, and C. L. Tsang, "Multiple Regression Models for Energy Use in Airconditioned Office Buildings in Different Climates," Energy Convers. Manag., pp. 2692-2697, 2010.

[4] M. Akole, M. Bongulwar, and B. Tyagi, "Predictive model of load and price for restructured power system using neural network," Int. Conf. Energy, Autom. Signal, pp. 1-6, 2011.

[5] J. Lei, H. Ning, and L. Yao, "A new method of loadshedding control on centrifugal water chiller sequencing," 2009 4th IEEE Conf. Ind. Electron. Appl., pp. 3204-3209, May 2009.

[6] P. Hsiao-Tien and T. Chung-Ming, "Modeling and forecasting the $\mathrm{CO} 2$ emissions, energy consumption, and economic growth in Brazil," 5th Conf. Sustain. Dev. Energy, Water Environ. Syst., vol. 36, no. 5, pp. 2315$3618,2011$.

[7] P. Monigatti, M. Apperley, and B. Rogers, "Power and Energy Visualization for the Micro-management of Household Electricity Consumption," Prococeedings AVI 2010, Rome, pp. 325-328, 2010

[8] Q. Cui, B. Luo, X. Huang, A. Dowhuszko, and J. Jiang, "Closed-form solution for minimizing power consumption in coordinated transmissions," EURASIP J. Wirel. Commun. Netw., vol. 2012, no. 1, p. 122, 2012.

[9] Q. Wang, X. Wang, and F. Xia, "Integration of Grey Model and Multiple Regression Model to Predict Energy Consumption," IEEE Proc., pp. 194-197, 2009.

[10] O. Kramer, B. Satzger, and J. Lassig, "Power Prediction in Smart Grids with Evolutionary Local Kernel Regression," HAIS Part 1 LNAI 6076, pp. 262-269, 2010.

[11] H. Chen, C. A. Canizares, and A. Singh, "ANN-based short-term load forecasting in electricity markets," 2001 IEEE Power Eng. Soc. Winter Meet. Conf. Proc. (Cat. No.01CH37194), vol. 2, no. 1, pp. 411-415, 2001. 